\title{
JURNAL GEOGRAFI
}
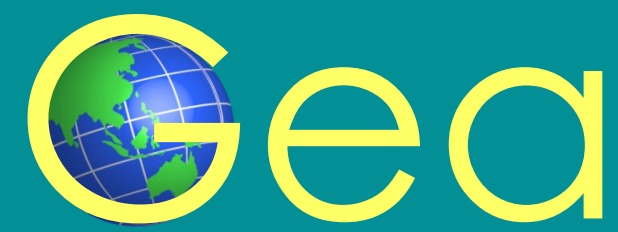

PERSEPSI GURU TERHADAP PENGUASAAN ADVANCE MATERIALS UNTUK PEMBELAJARAN GEOGRAFI Nandi, Hendro Murtianto, Totok Doyo Pamungkas, Indri Megantara Putri, Muhamad Akbar Wijaya

PERDAGANGAN BURUNG DI KOTA BANDUNG

(ANTARA EKONOMI, KEANEKARAGAMAN HAYATI, DAN KONSERVASI)

Asep Mulyadi, Moh. Dede

THE METHOD OF IMAGINARY MAP FOR HIGHER GEOGRAPHY LITERACY IN GENDER PERSPECTIVE Isma Yullia Rahma, Ahmad Yani, Bagja Waluya, Riki Ridwana

METODE DEFUZZIFIKASI ARTIFICIAL NEURAL NETWORK UNTUK KLASIFIKASI PENGGUNAAN LAHAN Harvini Wulansari

SINTESA GEOMORFOLOGI ANTROPOSEN KAWASAN CAGAR ALAM GEOLOGI KARANGSAMBUNG BAGIAN SELATAN

Puguh Dwi Raharjo, Eko Haryono

PERKEMBANGAN KAWASAN PERKOTAAN KECIL DI PINGGIRAN KOTA PEKALONGAN Fadjar Hari Mardiansjah, Paramita Rahayu

SKENARIO UNCERTAINTY JUMLAH PENAKAR CURAH HUJAN DI KOTA MAKASSAR Giarno, Muflihah, Mujahidin

ANALISIS RISIKO BANJIR TERHADAP FASILITAS PENDIDIKAN DI DKI JAKARTA Siti Dahlia, Fadiarman

ANALISIS PASCA BENCANA TANAH LONGSOR 1 JANUARI 2020 DAN EVALUASI PENATAAN KAWASAN DI KECAMATAN SUKAJAYA, KABUPATEN BOGOR

Heru Sri Naryanto, Firman Prawiradisastra, Ruki Ardiyanto, Wahyu Hidayat 


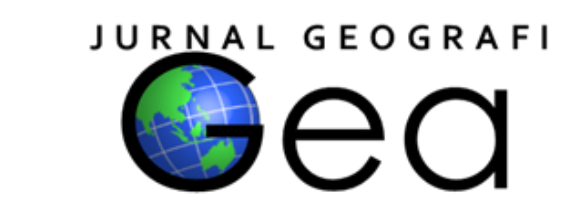

\section{DEWAN REDAKSI}

Penanggung Jawab

Dr. Ahmad Yani, M.Si.

Pimpinan Redaksi

Dr.rer.nat. Nandi, M.T., M.Sc

Ketua Pelaksana

Prof. Dr. Enok Maryani, M.S

\section{Dewan Editorial}

Bagja Waluya, M.Pd

Riki Ridwana, S.Pd., M.Sc

Totok Doyo Pamungkas, S.Si., M.Eng

\section{Mitra Bestari}

Prof. Dr. Ir. H. Dede Rohmat, M.T. (Universitas Pendidikan Indonesia)

Prof. Dr. H. Darsiharjo, M.S. (Universitas Pendidikan Indonesia)

Prof. Dr. H. Dede Sugandi, M.Si. (Universitas Pendidikan Indonesia)

Dr. Djoko Harmantyo, M.S. (Universitas Indonesia)

Dr. Muhammad Zid, M.Si. (Universitas Negeri Jakarta)

Dr. Epon Ningrum, M.Pd. (Universitas Pendidikan Indonesia)

Dr. Iwan Setiawan, M.Si. (Universitas Pendidikan Indonesia)

Dr. Lili Somantri, M.Si. (Universitas Pendidikan Indonesia) 


\section{DAFTAR ISI}

PERSEPSI GURU TERHADAP PENGUASAAN ADVANCE MATERIALS UNTUK PEMBELAJARAN GEOGRAFI

Nandi, Hendro Murtianto, Totok Doyo Pamungkas, Indri Megantara Putri,

Muhamad Akbar Wijaya 94-104

PERDAGANGAN BURUNG DI KOTA BANDUNG

(ANTARA EKONOMI, KEANEKARAGAMAN HAYATI, DAN KONSERVASI)

Asep Mulyadi, Moh. Dede 105-112

THE METHOD OF IMAGINARY MAP FOR HIGHER GEOGRAPHY LITERACY IN GENDER PERSPECTIVE

Isma Yullia Rahma, Ahmad Yani, Bagja Waluya, Riki Ridwana $113-119$

METODE DEFUZZIFIKASI ARTIFICIAL NEURAL NETWORK UNTUK KLASIFIKASI PENGGUNAAN LAHAN

Harvini Wulansari $120-140$

SINTESA GEOMORFOLOGI ANTROPOSEN KAWASAN CAGAR ALAM GEOLOGI KARANGSAMBUNG BAGIAN SELATAN

Puguh Dwi Raharjo, Eko Haryono .... $141-150$

PERKEMBANGAN KAWASAN PERKOTAAN KECIL DI PINGGIRAN KOTA PEKALONGAN

Fadjar Hari Mardiansjah, Paramita Rahayu $151-168$

SKENARIO UNCERTAINTY JUMLAH PENAKAR CURAH HUJAN DI KOTA MAKASSAR Giarno, Muflihah, Mujahidin 169-184

ANALISIS RISIKO BANJIR TERHADAP FASILITAS PENDIDIKAN DI DKI JAKARTA

Siti Dahlia, Fadiarman. 185-196

ANALISIS PASCA BENCANA TANAH LONGSOR 1 JANUARI 2020 DAN EVALUASI PENATAAN KAWASAN DI KECAMATAN SUKAJAYA, KABUPATEN BOGOR

Heru Sri Naryanto, Firman Prawiradisastra, Ruki Ardiyanto, Wahyu Hidayat. $197-213$ 

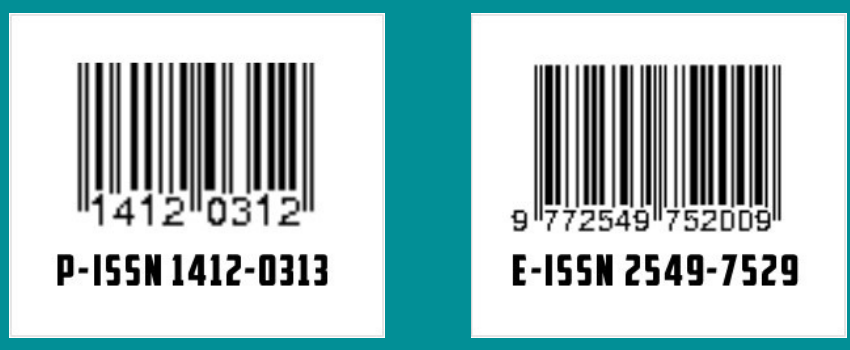